\title{
Determining the frequency of serious adverse reactions of inactive SARS-COV-2 vaccine
}

\author{
Ferit Kaya $^{\mathrm{a}}$ and Edibe Pirincci ${ }^{\mathrm{b}, *}$ \\ ${ }^{a}$ Department of Public Health, Faculty of Medicine, University ofAdiyaman, Adiyaman, Turkey \\ ${ }^{\mathrm{b}}$ Department of Public Health, Faculty of Medicine, University of Firat, Elazig, Turkey
}

Received 24 March 2021

Accepted 12 April 2021

\begin{abstract}
.
BACKGROUND: Vaccines are a new combat strategy against COVID-19. The success of a large number of vaccines and the continued development of vaccines will change the course of the current pandemic.

OBJECTIVE: The aim of the study was to determine the frequency of serious adverse reactions caused by the administration of inactive vaccine administration on healthcare workers during the COVID-19 pandemic.

METHODS: The prospective study was conducted among healthcare professionals working in city a Training and Research Hospital and applied to have the second dose of CoronaVac vaccine. The number of personnel included in the study were 329. The data were recorded in the SPSS 23 program and the chi-square test was used for statistical analysis.

RESULTS: The average age of the participants in the study was $35.77 \pm 9.07$. Of the participants, $28.1 \%$ were physicians. The frequency ratio of those who stated that they had serious adverse reactions after vaccination was $33.2 \%$. Three most common systemic serious adverse reactions were headache, state of sleep/fatigue, and nausea and vomiting respectively. Serious adverse reactions occurred within $1.14 \pm 04$ days after vaccine administration. The average duration of serious adverse reactions was determined as $1.68 \pm 0.77$ days. $62.2 \%$ of the participants with serious side effects were women $(p<0.001)$. Of the participants who had serious adverse reactions, $77.5 \%$ were health care professionals $(p<0.01)$.

CONCLUSION: No life-threatening serious adverse reaction was determined regarding the CoronaVac vaccine administered in this study. However, local serious adverse reactions, nausea/vomiting, fever and sleepiness/fatigue occurred frequently. Further studies are required on the newly introduced vaccine.
\end{abstract}

Keywords: Pandemic, healthcare professionals, fever, injection site, hospital, COVID-19

\section{Introduction}

The COVID-19 outbreak is a situation without an approved preparedness plan [1]. The number of confirmed COVID-19 pandemic cases reached 111.419.939 worldwide until March 2021. The number of people who lost their lives reached 2.470.772

*Address for correspondence: Prof. Dr. Edibe Pirincci, Department of Public Health Faculty of Medicine, Firat University, 23119, Elazig, Turkey. Tel.: +9 04242370000 / 6452; E-mail: edibepirincci@yahoo.com.
[2]. During the pandemic, some measures such as social distance, wearing a mask, ventilating the rooms, avoiding crowds, washing hands and closing the mouth while coughing reduce the risk of contact to the disease [3]. Tests, treatments and vaccines are being developed to combat this disease worldwide. Vaccines are a new combat strategy against COVID19. The success of a large number of vaccines and the continued development of vaccines will change the course of the current pandemic line [4]. 255 studies have been carried out worldwide against COVID-19, 
which are at the stage of preclinical or clinical development, as of February 25th, 2021 [4]. Vaccine safety is a major concern for the community and authorities [6]. While most people have previous experience with a safely administered vaccine, a small group may not be able to have the vaccine due to serious adverse reactions. Pain and redness on the injection site are common serious adverse reactions [7].

Various serious adverse reactions have been observed after the Pfizer-BioNTech COVID-19 vaccine. Local reaction was observed in $84.7 \%$ of the study group. In the same group, the incidence of at least one of the serious adverse reactions such as fever, headache, diarrhea, vomiting and shivering was reported to be $77.4 \%$ [8]. In the phase 1 and phase 2 studies conducted on the safety of the inactivated COVID-19 vaccine, the frequency of serious adverse reactions varied depending on the amount of dose administered and the duration of administration between two doses. As per the phase 2 results in the conducted study, the frequency of serious adverse reactions reported after inactivated COVID-19 vaccine was $19.0 \%$. The frequency of serious adverse reactions such as pain, itchiness and swelling was $14.3 \%$. The systemic effect frequency of the vaccine was documented as $7.1 \%$. No serious adverse reaction was stated in the same study [9].

The aim of the study was to determine the frequency of serious adverse reactions caused by the administration of inactive vaccine administration on healthcare workers during the COVID-19 pandemic.

\section{Methods}

\subsection{Study population}

This study was planned in a prospective type. The population of the study were healthcare professionals working in province in the southeast of Turkey (Adiyaman city). Training and Research Hospital and applied to have the second dose of CoronaVac vaccine. As of 25 January 2021, 3273 personnel are working in the relevant hospital and 2277 personnel have been vaccinated. As there is no accurate information about the serious adverse reaction occurrence ratio, with the 50\% prevalence, 5\% margin of error and $95 \%$ confidence interval $=\mathrm{Nt}^{2} \mathrm{pq}$ $/ \mathrm{d}^{2}(\mathrm{~N}-1)+\mathrm{t}^{2} \mathrm{pq}$ formula, the population of the personnel to be included in the study was determined as at least 329 individuals.

\subsection{Data collection}

The questionnaires were prepared after review of the evidence-based literature The questionnaire form was applied as face-to-face interviews by the researchers. Participation in the questionnaire was voluntary-basis and the questionnaires were administered after the participants were informed and signed the consent form.

\subsection{Statistical analysis}

The evaluation of the data obtained from the data collection form was made with the SPSS 23 package program. Means were provided with standard deviation. One of the nonparametric tests, $\mathrm{X}^{2}$ test was used. The results were evaluated at $95 \%$ confidence interval and $p<0.05$ was accepted as significant.

\section{Results}

Mean age of the participants in the study was $35.77 \pm 9.07$. Of the participants, $66.5 \%$ were married, $76.6 \%$ were university graduate, and $28.1 \%$ were doctors (Table 1). The frequency ratio of those who stated that they had serious adverse reactions after vaccination was $33.2 \% 1$. The frequency of those who had flu infection after the COVID-19 vaccine administration was $1.5 \%$ (Table 2). Three most common serious adverse reactions were headache, state of sleep/fatigue, pain and redness, respectively (Table 3). Serious adverse reactions occurred within $1.14 \pm 04$ days after vaccine administration. The average duration of serious adverse reactions was determined as $1.68 \pm 0.77$ days. Of the participants

Table 1

Distribution of sociodemographic characteristics

\begin{tabular}{llcc}
\hline $\begin{array}{l}\text { Sociodemographic } \\
\text { characteristics }\end{array}$ & & $n$ & $\%$ \\
\hline Gender & Male & 172 & 51.5 \\
& Female & 162 & 48.5 \\
Marital status & Married & 222 & 66.5 \\
& Single & 112 & 33.5 \\
Education level & Primary school graduate & 9 & 2.7 \\
& Secondary school graduate & 4 & 1.2 \\
& High school graduate & 65 & 19.5 \\
& University graduate & 256 & 76.6 \\
Profession & Doctor & 94 & 28.1 \\
& Nurse & 94 & 28.1 \\
& Health officer & 31 & 9.3 \\
& Other* & 115 & 34.4 \\
\hline
\end{tabular}

* Security guard, technician, data processing personnel, etc. 
experienced serious adverse reactions, $62.2 \%$ were female, and $37.8 \%$ were male. Serious adverse reactions were found to be significant as per the gender $(p<0.001)$. Serious adverse reaction frequency was $36.0 \%$ between $30-39$ age range. Of the participants with serious adverse reactions, $77.5 \%$ were health care professionals, while $22.5 \%$ non-medical staff $(p<0.01)$.

\section{Discussion}

At least one serious adverse reaction was stated on the $33.2 \%$ of the participants. In the phase 2 study of the inactivated vaccine developed against SARS-CoV-2 infection, the frequency of undesirable effects was found to be $19 \%$. The frequency rate of the participants who reported at least one local reaction after the administration of vaccine

Table 2

Distribution of post-vaccination period characteristics

\begin{tabular}{llcc}
\hline Post-vaccination period characteristics & & $n$ & $\%$ \\
\hline Have there been any serious adverse & Yes & 111 & 33.2 \\
reactions after vaccination? & No & 223 & 66.8 \\
Have you experienced an influenza in the & Yes & 5 & 1.5 \\
last one month? & No & 329 & 98.5 \\
\hline
\end{tabular}

produced by mRNA technology (Pfizer-BioNTech) was found to be $84.7 \%$. In the same study, systemic serious adverse reaction frequency was determined as $77.4 \% .{ }^{9}$ In the present study, it was determined that serious adverse reaction frequency was relatively low in the inactive vaccines, when compared with mRNA vaccines.

Local serious adverse reaction frequency was documented as $9.0 \%$. Of the local serious adverse reactions, $97.3 \%$ occurred in one day and $94.5 \%$ of the local serious adverse reactions last for a day or less. In phase 2 studies of the inactive vaccine developed against SARS-CoV-2 infection, the frequency of local side effects was found to be $15.5 \%$. In a study conducted, it was documented that local reactions occurring at the vaccine site were among the common serious adverse reactions [11].

Three most common systemic serious adverse reactions were headache, state of sleep/fatigue, and nausea and vomiting. The frequency of headache, which was one of the most common systemic serious adverse reactions, was found to be $16.8 \%$. Most of the headache complaints relapsed within the first day and more than half of the cases were found to be resolved within one day. Of the participants experienced headache, one fourth reported that their complaints lasted for at least three days. There was no

Table 3

Distribution of features regarding serious adverse reactions that occur after vaccination

\begin{tabular}{|c|c|c|c|c|c|c|c|}
\hline & \multirow[t]{2}{*}{ Symptoms } & \multicolumn{3}{|c|}{ Symptom onset } & \multicolumn{3}{|c|}{ Symptom progress } \\
\hline & & $\begin{array}{c}\text { In the } 1 \text { st day } \\
n(\%)\end{array}$ & $\begin{array}{c}\text { In the } 2 \text { nd day } \\
n(\%)\end{array}$ & $\begin{array}{c}\text { In the } 3 \text { rd and } \\
\text { following days } \\
n(\%)\end{array}$ & $\begin{array}{c}\text { In the } 1 \text { st day } \\
n(\%)\end{array}$ & $\begin{array}{c}\text { In the } 2 \text { nd day } \\
n(\%)\end{array}$ & $\begin{array}{c}\text { In the } 3 \text { rd and } \\
\text { following days } \\
n(\%)\end{array}$ \\
\hline Pain / redness / swelling & $30(9.0)$ & $29(97.3)$ & $1(3.3)$ & $0(0.0)$ & $15(94.5)$ & $9(2.7)$ & $6(1.8)$ \\
\hline Headache & $56(16.8)$ & $54(96.4)$ & $0(0.0)$ & $2(3.6)$ & $30(53.6)$ & $11(19.6)$ & $15(26.8)$ \\
\hline Fever & $12(3.6)$ & $10(83.4)$ & $1(8.3)$ & $1(8.3)$ & $7(58.3)$ & $1(8.3)$ & $4(33.4)$ \\
\hline State of sleep/fatigue & $46(13.8)$ & $41(89.1)$ & $3(6.5)$ & $2(4.3)$ & $25(54.3)$ & $12(26.1)$ & $9(19.6)$ \\
\hline Nausea/vomiting & $12(10.8)$ & $10(83.3)$ & $1(8.3)$ & $1(8.3)$ & $6(50.0)$ & $2(16.6)$ & $4(33.4)$ \\
\hline Allergy & $3(0.9)$ & $3(100)$ & $0(0.0)$ & $0(0.0)$ & $3(100.0)$ & $0(0.0)$ & $0(0.0)$ \\
\hline Extensive itchiness & $2(0.6)$ & $2(100.0)$ & $0(0.0$ & $0(0.0)$ & $2(100)$ & $0(0.0)$ & $0(0.0)$ \\
\hline Myalgia & $13(3.9)$ & $11(84.6)$ & $2(15.4)$ & $0(0.0$ & $2(15.4)$ & $4(30.8)$ & $7(53.8)$ \\
\hline *Other & $17(5.1)$ & $15(88.2)$ & $1(5.9)$ & $1(5.9)$ & $9(53.0)$ & $3(17.6)$ & $5(29.4)$ \\
\hline
\end{tabular}

*Tachycardia, loss of taste, feeling of throat swelling vertigo.

Table 4

Distribution of serious adverse reaction sociodemographic characteristics

\begin{tabular}{llccc}
\hline & & Yes & No & $p$ \\
\hline Gender & Male & $42(37.8)$ & $130(58.3)$ & $\chi^{2}=12.484$ \\
& Female & $69(62.2)$ & $93(41.7)$ & $p<0.001$ \\
Age & Under 30 & $32(28.8)$ & $61(27.4)$ & $\chi^{2}=0.08$ \\
& Between 30-39 & $40(36.0)$ & $83(37.2)$ & $p>0.05$ \\
\multirow{4}{*}{ Profession } & 40 and above & $39(35.1)$ & $79(35.4)$ & \\
& Health care worker & $86(77.5)$ & $138(61.9)$ & $\chi^{2}=8.159$ \\
& Non-health worker & $25(22.5)$ & $85(38.1)$ & $p<0.01$ \\
\hline
\end{tabular}


evidence that the inactive vaccine developed against SARS-CoV-2 caused headache [12].

In our study, the second most common adverse systemic effect was documented to be state of sleep and fatigue. It was found that most of the sleep fatigue complaints occur within a day and almost half of them last for at least a day. The inactivated vaccine developed against SARS-CoV-2 infection was found to cause fatigue with a frequency of $1.2 \%$ [9]. Due to the fact that the study was conducted among healthcare workers, intense working conditions might have affected the frequency of fatigue.

The third most common systemic serious adverse reaction was nausea and vomiting. The vast majority of nausea and vomiting complaints occurred within the first day and it was found that approximately one third of the individuals with complaints lasted three days or more. It was documented that the inactive vaccine developed against SARS-CoV-2 infection frequently caused nausea and vomiting complaints with a ratio of $1.2 \%$ [9].

The prevalence of fever, which was one of the serious adverse reactions experienced after vaccination, was found to be $3.6 \%$. Fever, another serious adverse reaction, was found to occur within a day in the majority of the participants and lasted for a day in more than half of the participants, and 3 days or more in one third of the participants. It was documented that the inactive vaccine developed against SARS-CoV-2 infection frequently caused nausea and vomiting with a rate of $2.4 \%$ [9].

Complaints of extensive itchiness, muscle pain, loss of taste, tachycardia, feeling of swelling in the throat and dizziness, which were the adverse side effects experienced after vaccination, were found to be less common than other serious adverse reactions.

It was determined that $1.5 \%$ of the participants had a flu infection in about a month after vaccination. This rate might have showed a difference depending upon the fact that the health care workers complied with the mask wearing rules.

The incidence of serious adverse reactions was found to be significantly higher among female participants when compared to male ones $(p<0.001)$. It was documented in a study conducted that post-vaccination hypertensive seizures occur more frequently among female participants than male ones [12]. Another study demonstrated that female and male participants gave different humoral responses to pathogens [13].

There was no significant difference between the frequency of serious adverse reactions and age groups among the participants $(p>0.05)$. This finding may be due to the fact that the study was conducted among the age group who have an active business life.

The frequency of serious adverse reactions experienced after vaccination was found to be significantly higher among health professionals compared to individuals working in support units $(p<0.01)$. Lifethreatening effects were not detected among the individuals who were administered the vaccine. No evidence was found regarding that the inactive vaccine developed against the SARS-CoV-2 infection has a life-threatening serious adverse reaction [9].

\section{Limitations}

The research was done in healthcare professionals working in province in the south east of the Turkey (Adiyaman city) Training and Research Hospital, the research cannot be generalized to the entire population. However, the sufficient number of samples is a strength of the research. Finally, the study depended on the self-evaluations of healthcare workers.

\section{Conclusion}

No life-threatening serious adverse reaction was determined in this study. Furthermore, local serious adverse reactions and nausea / vomiting, fever and sleepiness / fatigue occurred frequently. Further studies are required on this newly introduced vaccine.

\section{Conflict of interest}

The authors declare no potential conflicts of interest with respect to the research, authorship, and/or publication of this article.

\section{Funding}

The authors received no financial support for the research, authorship, and/or publication of this article.

\section{References}

[1] Irmak R. The most cited and co-cited COVID-19 articles: knowledge base for rehabilitation team members. Work. 2020;66(3):479-89. 
[2] WHO Coronavirus Disease (COVID-19) Dashboard | WHO Coronavirus Disease (COVID-19) Dashboard. Avaible from: https://covid19.who.int/. Accessed on: 01 March 2021.

[3] Advice for the public. Avaible from: https://www.who.int/ emergencies/diseases/novel-coronavirus.2019/advice-forpublic. Accessed on: 24 February 2021.

[4] COVID-19 vaccines. Avaible from: https://www.who.int/ emergencies/diseases/novel-coronavirus. Accessed on: 24 February 2021.

[5] Draft landscape and tracker of COVID-19 candidate vaccines. Avaible from: https://www.who.int/publications/ $\mathrm{m} /$ item/draft-landscape-of-covid-19-candidate-vaccines. 2019/advice-for-public. Accessed on: 24 February 2021.

[6] Johansen K, Nicoll A, Ciancio BC, Kramarz P. Pandemic influenza $\mathrm{A}(\mathrm{H} 1 \mathrm{~N} 1) 2009$ vaccines in the European Union. Euro Surveill. 2009;14:19361.

[7] Ask Us | Immunize BC. Avaible from: https://immuni zebc.ca/ask-us. Accessed on: 24 February 2021.

[8] Reactions and Adverse Events of the Pfizer-BioNTech COVID-19 Vaccine |CDC. Avaible from: https://www.cdc. gov/vaccines/covid-19/info-by-product/pfizer/reactogeni city.html. Accessed on: 22 February 2021.

[9] Shengli X, Kai D, Yuntao Z, Dongyang Z, Huajun Z, Zhiqiang X, Xinguo L, Cheng P, Yanbo Z, Wei Z, Yunkai Y, Wei C, Xiaoxiao G, Wangyang Y, Xuewei W, Zejun W,
Zhengli S, Yanxia W, Xuqin Y, Lianghao Z, Lili H, Qian W, Jia L, Yongli Y, Jing G, Wei Z, Xin W, Cong W, Wenhui W, Shihe H, Jianhui D, Ziyan M, An P, Zhiming Y, Shuo S, Wanshen G, Xiaoming Y. MD Effect of an inactivated vaccine against SARS-CoV-2 on safety and immunogenicity outcomes: interim analysis of 2 randomized clinical trials. JAMA. 2020;324:951-60.

[10] FDA. Vaccines and Related Biological Products Advisory Committee. December,10, 2020 Meeting Briefing Document- FDA. 2020. Available from: https://www.fda. gov/advisory-committees/advisory-committee-calendar/ vaccines-and-related-biological-products-advisorycommittee-december-10-2020-meeting-announcement\# event-information. Accessed on: 23 February 2021.

[11] Lombardi N, Crescioli G, Bettiol A, Tuccori M, Rossi M, Bonaiuti R, Ravaldi C, Levi M, Mugelli A, Ricci S, Lippi F, Azzari C, Bonanni P, Vannacci A. Vaccines safety in children and in general population: A pharmacovigilance study on adverse events following antiinfective vaccination in Italy. Front Pharmacol. 2019; 10:948.

[12] Griffioen M, Halsey N. Gender differences in immediate hypersensitivity reactions to vaccines: A review of the literature. Public Health Nurs. 2014;31:206-14.

[13] Whitacre CC. Sex differences in autoimmune disease. Nat Immunol. 2001;2:777-80. 